\title{
Successful Treatment with Ensartinib After Alectinib- induced Hyperbilirubinemia in ALK-Positive NSCLC
}

\author{
Ling Peng $\left.\mathbb{( D}^{1}\right)^{\prime}$ \\ Kui $\mathrm{Xiao}^{2, *}$ \\ Jian $\mathrm{Cui}^{3}$ \\ Xiang-Hua $\mathrm{Ye}^{4}$ \\ Yong-Chang Zhang $\mathbb{D}^{5}$ \\ Li Mao ${ }^{6}$ \\ Giovanni Selvaggi (1D ${ }^{7}$ \\ Jennifer Yen $^{8}$ \\ Justin Stebbing $\mathbb{D}^{9}$ \\ 'Department of Pulmonary and Critical \\ Care Medicine, Zhejiang Provincial People's \\ Hospital, Hangzhou, Zhejiang Province, \\ People's Republic of China; ${ }^{2}$ Department of \\ Pulmonary and Critical Care Medicine, The \\ Second Xiangya Hospital, Central South \\ University, Changsha, Hunan Province, \\ People's Republic of China; ${ }^{3}$ Shanghai \\ BioGenius Bioinformatics Institute, Shanghai, \\ People's Republic of China; ${ }^{4}$ Department of \\ Radiotherapy, The First Affiliated Hospital, \\ School of Medicine, Zhejiang University, \\ Hangzhou, Zhejiang Province, People's \\ Republic of China; ${ }^{5}$ Department of Medical \\ Oncology, Lung Cancer and Gastrointestinal \\ Unit, Hunan Cancer Hospital, The Affiliated \\ Cancer Hospital of Xiangya School of \\ Medicine, Changsha, Hunan, People's \\ Republic of China; ${ }^{6}$ Betta Pharmaceuticals, \\ Hangzhou, People's Republic of China; \\ ${ }^{7}$ X-Covery Holdings, Palm Beach Gardens, \\ FL, USA; ${ }^{8}$ Guardant Health, Inc., Redwood \\ City, CA, USA; ${ }^{9}$ Division of Cancer, \\ Department of Surgery and Cancer, Imperial \\ College London, London, UK
}

*These authors contributed equally to this work

Correspondence: Ling Peng

Department of Pulmonary and Critical

Care Medicine, Zhejiang Provincial

People's Hospital, No. 158 Shangtang

Road, Hangzhou, Zhejiang Province,

People's Republic of China

Tel/Fax +86-57I-85893509

Email drpengling@hotmai.com
Background: Alectinib is approved for the treatment of advanced non-small-cell lung cancer (NSCLC) harboring ALK rearrangements. Although generally well tolerated, alectinib can cause serious or life-threatening side effects.

Case Presentation: Here, we report a case of a patient with NSCLC with an EML4-ALK fusion and was treated with alectinib but who developed grade 4 hyperbilirubinemia after five months on therapy. Alectinib was discontinued, and an artificial liver support system (ALSS) was used with an impressive decline in bilirubin levels. After two months drug-free, the patient experienced disease progression. Ensartinib was initiated as second-line treatment with a best response of stable disease after three months of therapy with no evidence of hyperbilirubinemia.

Conclusion: This is the first report of ensartinib treatment after alectinib-induced hyperbilirubinemia which was successfully relieved by ALSS treatment and targeted drug cessation. Keywords: ensartinib, alectinib, hyperbilirubinemia, NSCLC, ALK

\section{Introduction}

ALK-positive non-small-cell lung cancer (NSCLC) is identified in $3-7 \%$ of advanced NSCLC cases. ${ }^{1}$ First generation ALK tyrosine kinase inhibitor (TKI) crizotinib has been the standard of care for the past 10 years. ${ }^{2}$ Second-generation ALK inhibitors such as alectinib were developed to be more effective than crizotinib especially in targeting brain metastases. Based on two large randomized clinical trials, ALEX $^{3}$ and J-ALEX, ${ }^{4}$ alectinib was approved for the first-line treatment of ALK-positive NSCLC. There are differences in the toxicity profiles between different ALK inhibitors with typically gastrointestinal and hepatic toxicities with ceritinib, visual disorders with crizotinib, edema, myalgias and dysgeusia with alectinib, and respiratory complications with brigatinib. ${ }^{5}$ These are possibly due to off-target effects inherent to the molecular structure of each ALK TKI.

Alectinib is generally well tolerated, but some more common adverse events (AEs) are observed including anemia, myalgia, and fatigue among others. ${ }^{6}$ However, the most common of these (all grades) is increased blood bilirubin $(36.2 \%){ }^{7}$ Here we present a case of an advanced stage NSCLC patient with a variant 2 EML4-ALK fusion who developed a severe toxicity of hyperbilirubinemia secondary to alectinib. ALSS was used twice in his treatment with other hepatoprotective therapies, and a therapeutic switch to ensartinib led to stable disease without liver toxicity and with normal bilirubin levels. We present the following case in accordance with the CARE reporting checklist. 


\section{Case Presentation}

A 56-year-old Chinese male smoker was diagnosed with stage IV NSCLC in December 2019. His chest CT scan (Figure 1A and B) revealed a mass in the right lower lobe and right-side pleural effusion. PET-CT
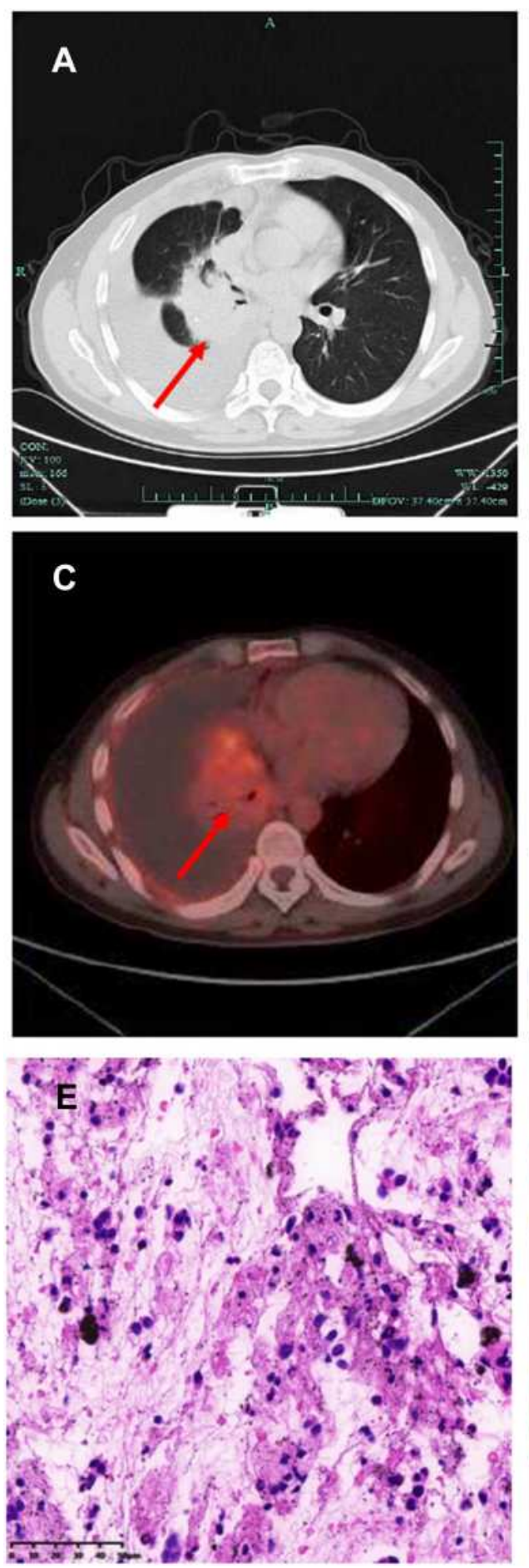

indicated a mass in the right lower hilar region with an elevated standardized uptake value (SUV) (Figure 1C), multiple lymph node enlargement, and bone metastases (Figure 1D). A diagnosis of lung adenocarcinoma was made on endobronchial ultrasound-guided
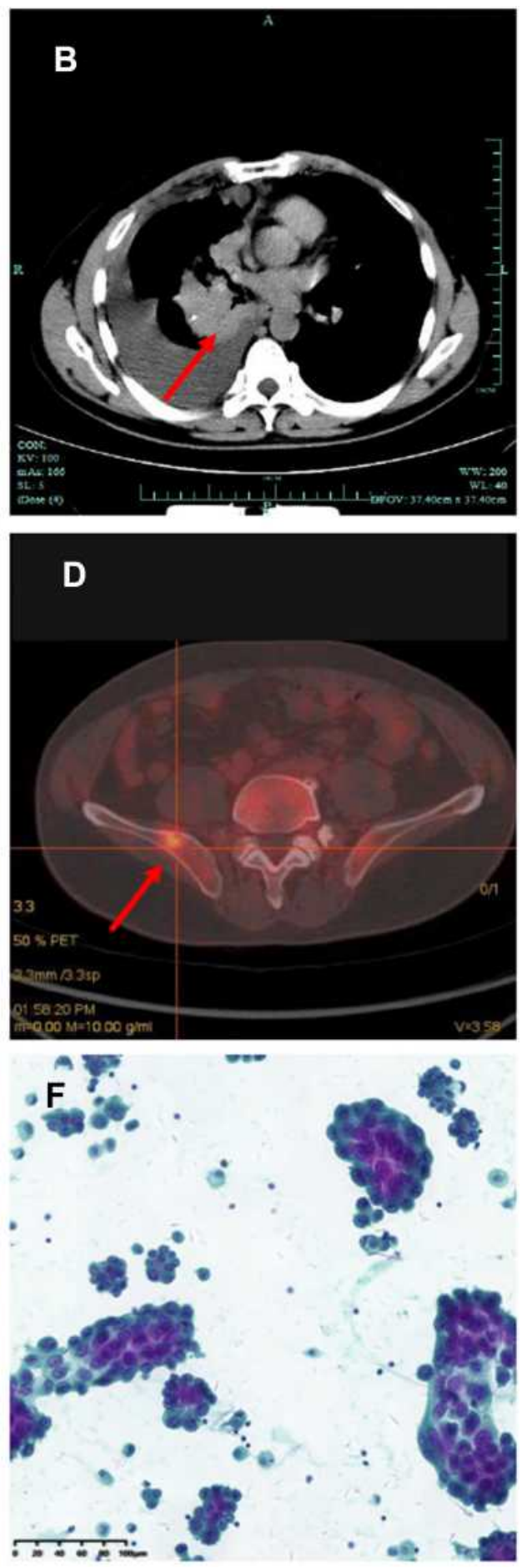

Figure I Diagnoses of right lower lung adenocarcinoma, stage IV. (A) CT scan lung on Dec 17, 2019; (B) CT mediastinum on Dec I7, 20I9; (C and D) PET-CT scan on Dec 3I, 2019; (E) Histopathologic findings of lung adenocarcinoma of mediastinal lymph node from EBUS-TBNA (H\&E, 40x). (F) Cytology from pleural effusion. Significant abnormal findings of right lung, lymph node and bone metastases were noted (arrow). 
transbronchial needle aspiration (TBNA-EBUS) of lymph nodes (Figure 1E). Lung adenocarcinoma cells were also found in pleural effusion samples (Figure $1 F)$. Next generation sequencing (NGS) of peripheral blood ctDNA indicated $E M L 4-A L K$, variant 2 (E20-
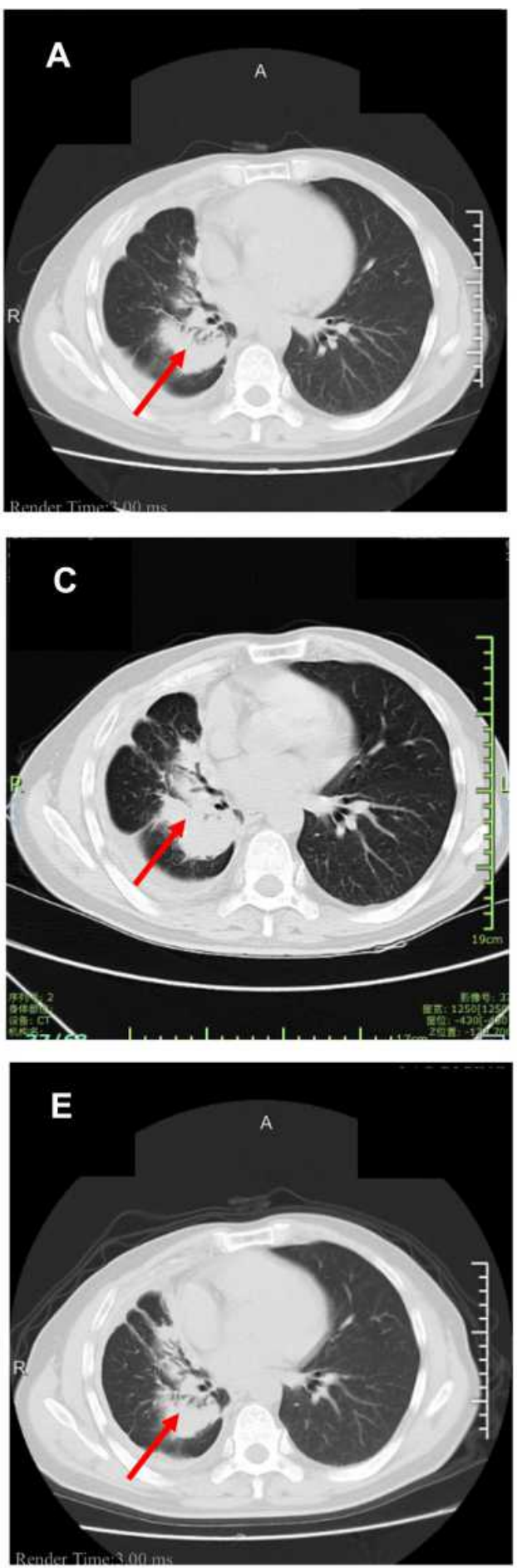

A20). Alectinib was given orally, at a dose of $600 \mathrm{mg}$ twice daily from January 7, 2020, which resulted in partial response and patient continued to respond after four months of treatment (Figure 2A and $\mathrm{B}$ ). He was noted to have an increased total
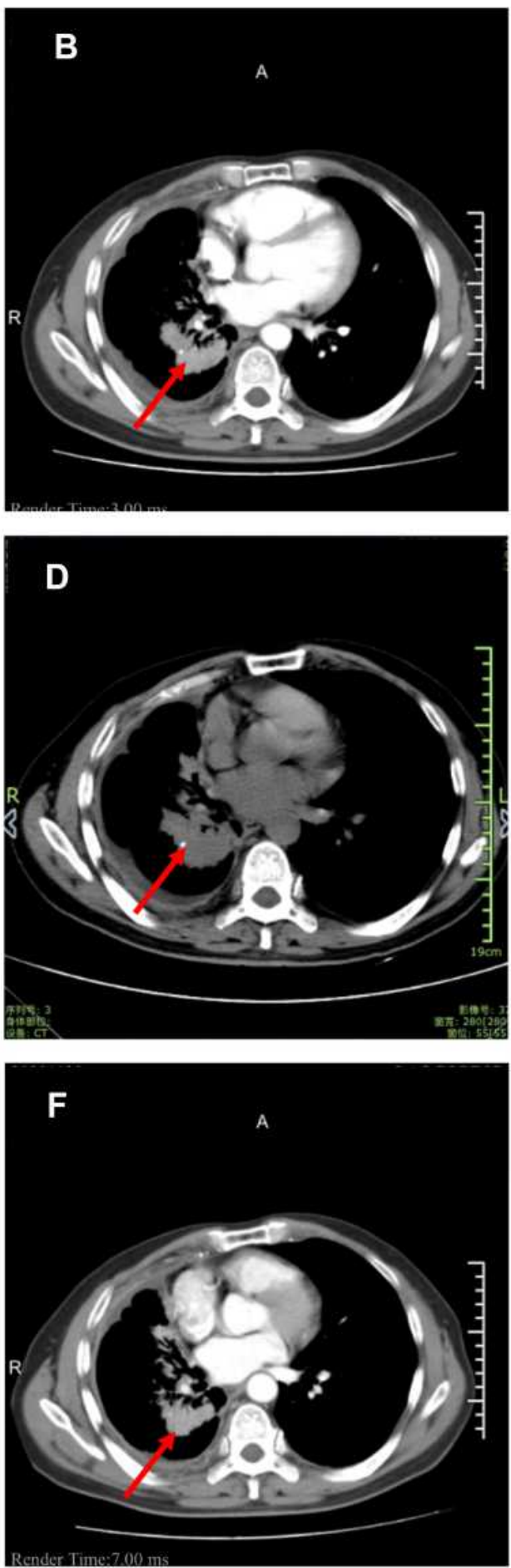

Figure 2 CT scans before and after ensartinib. (A) CT lung captured on May 6, 2020; (B) CT mediastinum on May 6, 2020; (C) CT lung on Aug 5, 2020 (before ensartinib); (D) CT mediastinum on Aug 5, 2020 (before ensartinib); (E) CT on Nov 30, 2020 (after ensartinib); (F) CT on Nov $30 \mathrm{~h}, 2020$ (after ensartinib). Significant abnormal findings of right lung were noted (arrow). 
bilirubin (TBIL) of $59.2 \mu \mathrm{mol} / \mathrm{L}$ on May 5, 2020 (on alectinib for 119 days) but did not refer to the doctor.

On May 29, 2020, the patient presented with jaundice, pruritus and fatigue. His Karnofsky performance status (KPS) rating fell to 60 points. The laboratory result showed TBIL $315.0 \mu \mathrm{mol} / \mathrm{L}$, DBIL (direct bilirubin) $260.7 \mu \mathrm{mol} / \mathrm{L}$, and IBIL (indirect bilirubin) 54.3 $\mu \mathrm{mol} / \mathrm{L}$. Serum ALP (alkaline phosphatase) was $374 \mathrm{U} /$ L, total bile acid $179.9 \mu \mathrm{mol} / \mathrm{L}$, GGT (gamma-glutamyl transferase) $47 \mathrm{U} / \mathrm{L}$ and aminotransferase levels were AST $34 \mathrm{U} / \mathrm{L}$ and ALT $42 \mathrm{U} / \mathrm{L}$. Therefore, a diagnosis of alectinib-induced grade 4 hyperbilirubinemia $(>10.0 \times \mathrm{ULN}$ if baseline was normal, Common Terminology Criteria for Adverse Events, v.5.0) was considered. Alectinib was held due to hepatotoxicity. Of note, he had no known liver metastases at baseline nor at follow-up scans. Assessment for viral hepatitis was unrevealing.

After hepatoprotective treatment comprising ursodeoxycholic acid, glycyrrhizin and glutathione was administered for one week, his bilirubin levels were still worsening, while AST and ALT levels remained similar. Drug-induced liver failure was diagnosed. Although artificial liver support or liver transplantation were both therapeutic choices for liver failure, considering he was a patient with advanced cancer, ALSS was an optimal choice. He received ALSS using a double plasma molecular absorption system (DPMAS) on June 5 and June 8, 2020, for the severe liver dysfunction. The total bilirubin continuously decreased after two courses of ALSS (Figure 3).

Alectinib was, therefore, discontinued for more than two months when a chest CT scan on August 5, 2020 revealed enlargement of lung lesions (Figure 2C and D) and overall disease progression. Ensartinib $225 \mathrm{mg}$ once daily was given orally from August 29 as a second-line ALK TKI with initial tumor shrinkage after three months (Figure 2E and F) and stable disease overall by RECIST (Response Evaluation Criteria in Solid Tumors) criteria version 1.1. Toxicity of second-line ensartinib was grade 1 ALT and AST elevation and grade 1 skin rash, with no abnormality of bilirubin. The timeline of this patient is summarized in Figure 4.

\section{Discussion}

Several ALK TKIs have been developed and approved by the US Food and Drug Administration (FDA) and/or the European Medicines Agency (EMA) for the treatment of

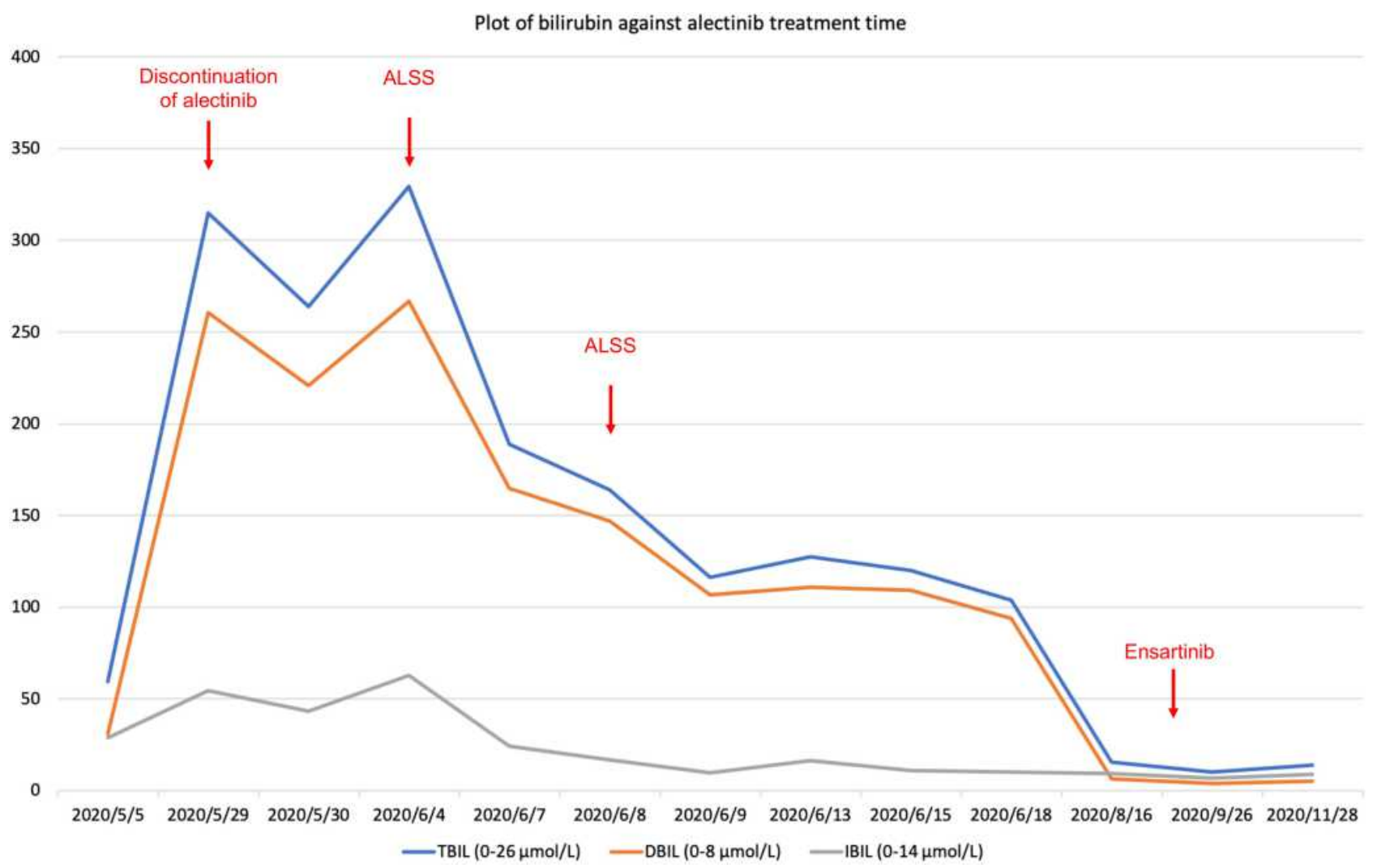

Figure 3 Bilirubin levels of this patient. 


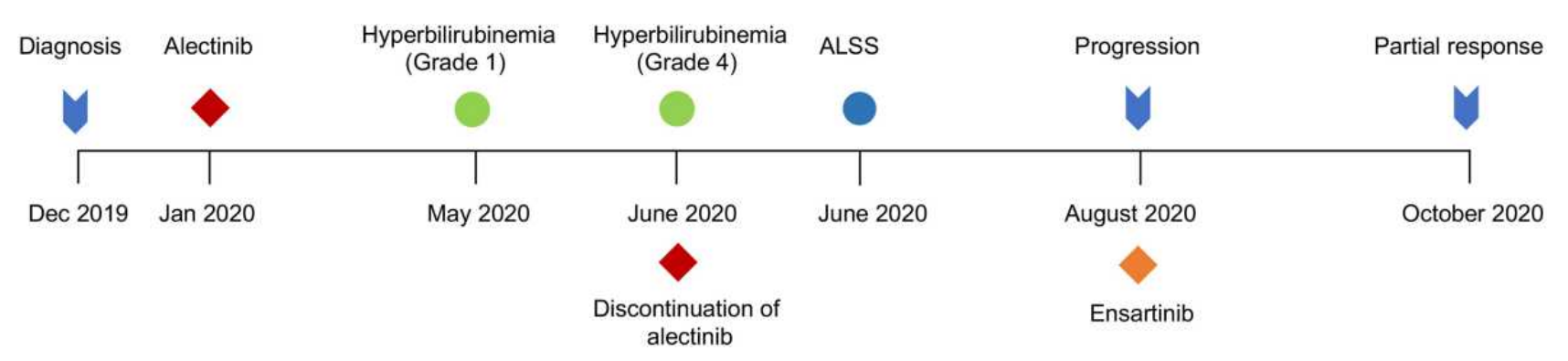

Figure 4 Timeline of patient history.

ALK-positive advanced NSCLC, including crizotinib, ceritinib, alectinib, brigatinib, and lorlatinib. The toxicity profile of different ALK TKIs is different due to their different structure and the ensuing off-target effects. In fact, alectinib is associated with a relatively high incidence of hepatotoxicity, while brigatinib is associated with a unique pulmonary toxicity and lorlatinib exhibits a peculiar neurotoxicity. Alectinib is predominantly metabolized into M4, and M4 is metabolized into M6by CYP3A4. ${ }^{8}$ Alectinib is not a substrate for transporters, coadministration with transporter inhibitors or inducers are not expected to have relevant implication.

Liver and kidney are the two major organs for the elimination of ALK TKIs. Liver elimination represents the major clearance pathway, with minor contributions from renal elimination. Hepatotoxicity of ALK TKI has been evaluated and compared in a meta-analysis. ${ }^{9}$ Severe ALK inhibitor-induced hepatotoxicity has previously been reported. ${ }^{10,11}$ Druginduced liver injury (DILI) can be divided into three main categories based on biochemical injury patterns: hepatocellular, cholestatic, and mixed. ${ }^{12}$ Most cases of drug-induced structural hepatotoxicity can be either acute or chronic, involving histological features of hepatocellular damage, cholestasis and/or steatosis. Histologic analysis of severe acute hepatitis of a patient receiving alectinib revealed inflammatory infiltrate and ductular proliferation connecting portal tracts and centrilobular areas. ${ }^{11}$ Hyperbilirubinemia is not a class-specific toxicity and seen specifically with alectinib and so not typical for other ALK TKIs.

Mechanisms of TKI-induced toxicity was studied in human hepatocyte cell lines, indicating mitochondrial toxicity and inhibition of glycolysis might relate to hepatotoxicity of TKI drugs. ${ }^{13}$ ROS-dependent DNA damage was found to be a possible mechanism of ALK inhibitorinduced hepatotoxicity. ${ }^{14}$

Adverse drug reactions that led to discontinuation of alectinib due to hyperbilirubinemia was reported as $1.3 \%$ in trials. As per the package insert, for grade 3 hyperbilirubinemia, alectinib should be temporarily withheld until recovery to baseline or to less than or equal to 1.5 times ULN (upper limits of normal). The resolution pattern of hepatotoxicity varies greatly. ${ }^{10}$ In our case, jaundice did not resolve after discontinuation of alectinib for seven days. Although ALSS has not received approval for routine use for the most common causes of hepatic failure, we used it here to improve hepatic function. ${ }^{12}$ This patient experienced grade 4 hyperbilirubinemia, therefore, permanent discontinuation of alectinib was advised.

We also identified an EML4-ALK variant 2 (EML4-ALK fusion on exon 20) in the patient's peripheral blood ctDNA by NGS. EML4-ALK variant 2 accounts for about $10 \%$ of $E M L 4-A L K$ variants. ${ }^{15}$ Different variants may respond largely differently toward ALK TKIs, as variant 3a- or 5aharboring cells were resistant to ALK inhibitor with $>10$-fold higher half maximal inhibitory concentration in vitro. ${ }^{16}$ Clinical data also demonstrated different ALK variants have different clinical response to ALK TKIs. ${ }^{17}$ However, it is unknown whether the variant type of $A L K$ fusion has an impact on toxicity. Analysis of plasma samples obtained postprogression on alectinib from pooled analysis of two prospective trials revealed that 53\% samples harbored 16 distinct ALK mutations, ${ }^{18}$ which may guide alectinibresistant treatments. Although this patient developed disease progression, we speculate that this was more likely due to discontinuation of alectinib than resistance to alectinib. For this reason, we did not elect to perform tissue or liquid biopsy sequencing to investigate presence of resistance mutations at later time points.

Previous research has provided evidence of a crucial role for the adaptive immune system in the pathogenesis of DILI. ${ }^{19}$ HLA-restricted T cell immune responses have been investigated using lymphocytes from patients with DILI. ${ }^{20}$ We performed whole-genome sequencing (WGS) from peripheral blood of this patient. Pathway analysis of SNVs 
(single nucleotide variants) from WGS of the patient's peripheral blood indicated enrichment of genes involved in many biological functions, including antigen presentation (Supplementary Methods, Tables S1-S3). No definitive gene variants were discovered to potentially explain alectinib-induced hyperbilirubinemia for this patient.

The subsequent treatment options for intolerance to an ALK inhibitor include switching to another ALK inhibitor or chemotherapy. A previous report described a patient with a crizotinib-induced hepatitis then treated with ceritinib salvage treatment, which indicated switch from one ALK TKI to another one would be a feasible and safe choice. ${ }^{21}$ Phase 1 and 2 clinical trials have shown that ensartinib is effective for patients whose cancer has progressed on crizotinib as well as second-generation ALK inhibitors. Ensartinib was approved on November 17, 2020 by the National Medical Products Administration (NMPA) of China for the second-line treatment of ALKpositive NSCLC after progression or intolerance of crizotinib based on the single-arm Phase 2 trial. ${ }^{22}$ In addition, a recently reported Phase 3 trial, eXalt3, investigated ensartinib compared with crizotinib in the first-line setting and showed a statistically significant longer PFS for ensartinib. ${ }^{23}$ Furthermore, in patients without measurable brain metastases at baseline, the 12-month relapse rate was much lower for patients given ensartinib compared with crizotinib $(\mathrm{HR}=0.32 ; P=0.0001),{ }^{23}$ indicating the intracranially prophylactic role of ensartinib. Considering the drug label and accessibility, ensartinib might be a reasonable treatment strategy for patients who are intolerant of alectinib as we showed in this case, with clinical benefit and no recurrence of severe hepatotoxicity.

\section{Conclusion}

To the best of our knowledge, this is the first report of grade 4 hyperbilirubinemia in a patient treated with alectinib, followed by ensartinib. Alectinib-induced hyperbilirubinemia of this patient was successfully managed with ALSS, suggesting that the latter may be an adjunct to drug cessation. Close monitoring of liver function remains a mandatory requirement for patients receiving ALK TKIs regardless of line of therapy and duration of treatment.

\section{Data Sharing Statement}

All relevant data and diagnostic results are contained. The raw data is not made available in consideration of confidentiality.

\section{Ethics Approval and Consent to Participate}

This study has been approved by Ethic Committee of Zhejiang Provincial People's Hospital. The patient signed an informed consent.

\section{Consent for Publication}

The patient and his family were informed that the information published may potentially compromise anonymity. Publication was consented by the patient. Written informed consent was obtained from the case patient for publication of this report and any accompanying images. A copy of the written consent is available for review by the Editor of this journal.

\section{Acknowledgments}

The authors would like to thank the patient's family for giving consent and for providing the detail information of this case.

\section{Author Contributions}

All authors made a significant contribution to the work reported, whether that is in the conception, study design, execution, acquisition of data, analysis and interpretation, or in all these areas; took part in drafting, revising or critically reviewing the article; gave final approval of the version to be published; have agreed on the journal to which the article has been submitted; and agree to be accountable for all aspects of the work.

\section{Funding}

This study was partially supported by Natural Science Foundation of Zhejiang Province, China (grant number: LY19H160041).

\section{Disclosure}

JS' conflicts can be found at https://www.nature.com/onc/ editors. None are relevant to this work. He is also the Editor in Chief for Oncogene. LM is an employee of Betta Pharmaceuticals. GS is employed by, and holds stock in, X-covery Holdings, Inc. JY is an employee and stockholder of Guardant Health. The authors report no other conflicts of interest in this work.

\section{References}

1. Roskoski R Jr. Anaplastic lymphoma kinase (ALK) inhibitors in the treatment of ALK-driven lung cancers. Pharmacol Res. 2017;1 17:343-356. doi:10.1016/j.phrs.2017.01.007 
2. Kazandjian D, Blumenthal GM, Chen HY, et al. FDA approval summary: crizotinib for the treatment of metastatic non-small cell lung cancer with anaplastic lymphoma kinase rearrangements. Oncologist. 2014;19:e5-11. doi:10.1634/theoncologist.2014-0241

3. Peters S, Camidge DR, Shaw AT, et al. Alectinib versus crizotinib in untreated ALK-positive non-small-cell lung cancer. $N$ Engl J Med. 2017;377:829-838. doi:10.1056/NEJMoa1704795

4. Hida T, Nokihara H, Kondo M, et al. Alectinib versus crizotinib in patients with ALK-positive non-small-cell lung cancer (J-ALEX): an open-label, randomised phase 3 trial. Lancet. 2017;390:29-39. doi:10.1016/S0140-6736(17)30565-2

5. Kassem L, Shohdy KS, Lasheen S, Abdel-Rahman O, Ali A, AbdelMalek RR. Safety issues with the ALK inhibitors in the treatment of NSCLC: a systematic review. Crit Rev Oncol Hematol. 2019;134:56-64. doi:10.1016/j.critrevonc.2018.11.004

6. Breadner D, Blanchette P, Shanmuganathan S, Boldt RG, Raphael J. Efficacy and safety of ALK inhibitors in ALK-rearranged non-small cell lung cancer: a systematic review and meta-analysis. Lung Cancer. 2020;144:57-63. doi:10.1016/j.lungcan.2020.04.011

7. Tamura T, Kiura K, Seto T, et al. Three-year follow-up of an alectinib phase I/II study in ALK-positive non-small-cell lung cancer: AF-001JP. J Clin Oncol. 2017;35:1515-1521. doi:10.1200/JCO.20 16.70 .5749

8. Zhao D, Chen J, Chu M, Long X, Wang J. Pharmacokinetic-based drug-drug interactions with anaplastic lymphoma kinase inhibitors: a review. Drug Des Devel Ther. 2020;14:1663-1681. doi:10.2147/ DDDT.S249098

9. Liu B, Yuan M, Sun Y, et al. Incidence and risk of hepatic toxicities associated with anaplastic lymphoma kinase inhibitors in the treatment of non-small-cell lung cancer: a systematic review and meta-analysis. Oncotarget. 2018;9(10):9480-9488. doi:10.18632/ oncotarget. 23840

10. Charville GW, Padda SK, Sibley RK, Puthillath A, Kwo PY. Resolution of crizotinib-associated fulminant hepatitis following cessation of treatment. Case Rep Hepatol. 2018;2018:3413592. doi: $10.1155 / 2018 / 3413592$

11. Zhu VW, Lu Y, Ou SI. Severe acute hepatitis in a patient receiving alectinib for ALK-positive non-small-cell lung cancer: histologic analysis. Clin Lung Cancer. 2019;20:e77-e80. doi:10.1016/j. cllc.2018.09.006

12. Gonzalez HC, Jafri SM, Gordon SC. Management of acute hepatotoxicity including medical agents and liver support systems. Clin Liver Dis. 2017;21:163-180. doi:10.1016/j.cld.2016.08.012
13. Mingard C, Paech F, Bouitbir J, Krahenbuhl S. Mechanisms of toxicity associated with six tyrosine kinase inhibitors in human hepatocyte cell lines. J Appl Toxicol. 2018;38(3):418-431. doi:10.1002/ jat.3551

14. Yan H, Du J, Chen X, et al. ROS-dependent DNA damage contributes to crizotinib-induced hepatotoxicity via the apoptotic pathway. Toxicol Appl Pharmacol. 2019;383:114768. doi:10.1016/j.taap.20 19.114768

15. Sabir SR, Yeoh S, Jackson G, Bayliss R. EML4-ALK Variants: biological and molecular properties, and the implications for patients. Cancers. 2017;9. doi:10.3390/cancers9090118

16. Woo CG, Seo S, Kim SW, et al. Differential protein stability and clinical responses of EML4-ALK fusion variants to various ALK inhibitors in advanced ALK -rearranged non-small cell lung cancer. Ann Oncol. 2017;28:mdw693. doi:10.1093/annonc/mdw693

17. Tu H-Y, Zhang Y-C, Wu Y-L. A story of ALK variants and the efficacy of ALK inhibitors: moving toward precision oncology. Transl Cancer Res. 2017;6:S292-S295. doi:10.21037/tcr.2017.03.07

18. Noe J, Lovejoy A, Ou SI, et al. ALK mutation status before and after alectinib treatment in locally advanced or metastatic ALK-positive NSCLC: pooled analysis of two prospective trials. J Thorac Oncol. 2020;15:601-608. doi:10.1016/j.jtho.2019.10.015

19. Grove J, Aithal G. Human leukocyte antigen genetic risk factors of drug-induced liver toxicology. Expert Opin Drug Metab Toxicol. 2014;11:1-15.

20. Ali S-E, Waddington J, Park K, Meng X. Definition of the chemical and immunological Signals involved in drug-induced liver injury. Chem Res Toxicol. 2019;33(1):61-76. doi:10.1021/acs.chemrestox. $9 \mathrm{~b} 00275$

21. Sassier M, Mennecier B, Gschwend A, et al. Successful treatment with ceritinib after crizotinib induced hepatitis. Lung Cancer. 2016;95:15-16. doi:10.1016/j.lungcan.2016.02.008

22. Yang Y, Zhou J, Zhou J, et al. Efficacy, safety, and biomarker analysis of ensartinib in crizotinib-resistant, ALK-positive non-small-cell lung cancer: a multicentre, phase 2 trial. Lancet Respir Med. 2020;8:45-53. doi:10.1016/S2213-2600(19)30252-8

23. Selvaggi G, Wakelee HA, Mok T, et al. Phase III randomized study of ensartinib vs crizotinib in anaplastic lymphoma kinase (ALK) POSITIVE NSCLC patients: eXalt3. J Thoracic Oncol. 2020;15: e41-e42. doi:10.1016/j.jtho.2020.08.003
OncoTargets and Therapy

\section{Publish your work in this journal}

OncoTargets and Therapy is an international, peer-reviewed, open access journal focusing on the pathological basis of all cancers, potential targets for therapy and treatment protocols employed to improve the management of cancer patients. The journal also focuses on the impact of management programs and new therapeutic agents and protocols on patient perspectives such as quality of life, adherence and satisfaction. The manuscript management system is completely online and includes a very quick and fair peer-review system, which is all easy to use. Visit http://www.dovepress.com/ testimonials.php to read real quotes from published authors. 